\title{
The effective temperature scale of M dwarfs ${ }^{\star}$
}

\author{
A. S. Rajpurohit ${ }^{1}$, C. Reylé ${ }^{1}$, F. Allard ${ }^{2}$, D. Homeier ${ }^{2}$, M. Schultheis ${ }^{1,3}$, M. S. Bessell ${ }^{4}$, and A. C. Robin ${ }^{1}$ \\ ${ }^{1}$ Institut UTINAM CNRS 6213, Observatoire des Sciences de l'Univers THETA Franche-Comté-Bourgogne, \\ Université de Franche-Comté, Observatoire de Besançon, BP 1615, 25010 Besançon Cedex, France \\ e-mail: arvind@obs-besancon.fr \\ 2 Centre de Recherche Astrophysique de Lyon, UMR 5574: CNRS, Université de Lyon, École Normale Supérieure de Lyon, \\ 46 allée d'Italie, 69364 Lyon Cedex 7, France \\ ${ }^{3}$ Université de Nice Sophia-Antipolis, CNRS, Observatoire de Côte d'Azur, Laboratoire Cassiopée, 06304 Nice Cedex 4, France \\ ${ }^{4}$ Research School of Astronomy and Astrophysics, Mount Stromlo Observatory, Cotter Road, Weston Creek, ACT 2611, Australia
}

Received 22 February 2013 / Accepted 12 April 2013

\section{ABSTRACT}

Context. Despite their large number in the Galaxy, $\mathrm{M}$ dwarfs remain elusive objects and the modeling of their photosphere has long remained a challenge (molecular opacities, dust cloud formation).

Aims. Our objectives are to validate the BT-Settl model atmospheres, update the $\mathrm{M}$ dwarf $T_{\text {eff }}$-spectral type relation, and find the atmospheric parameters of the stars in our sample.

Methods. We compare two samples of optical spectra covering the whole M dwarf sequence with the most recent BT-Settl synthetic spectra and use a $\chi^{2}$ minimization technique to determine $T_{\text {eff. }}$. The first sample consists of 97 low-resolution spectra obtained with New Technology Telescope (NTT) at La Silla Observatory. The second sample contains 55 medium-resolution spectra obtained at the Siding Spring Observatory (SSO). The spectral typing is realized by comparison with already classified M dwarfs.

Results. We show that the BT-Settl synthetic spectra reproduce the slope of the spectral energy distribution and most of its features. Only the $\mathrm{CaOH}$ band at $5570 \AA$ and $\mathrm{AlH}$ and $\mathrm{NaH}$ hydrides in the blue part of the spectra are still missing in the models. The $T_{\text {eff }}$ scale obtained with the higher resolved SSO $2.3 \mathrm{~m}$ spectra is consistent with that obtained with the NTT spectra. We compare our $T_{\text {eff }}$ scale with those of other authors and with published isochrones using the BT-Settl colors. We also present relations between effective temperature, spectral type, and colors of the M dwarfs.

Key words. stars: atmospheres - stars: low-mass - stars: fundamental parameters

\section{Introduction}

Low-mass stars of less than $1 M_{\odot}$ are the dominant stellar component of the Milky Way. They constitute $70 \%$ of all stars (Reid \& Gizis 1997; Bochanski et al. 2010) and 40\% of the total stellar mass of the Galaxy (Chabrier 2003, 2005). Our understanding of the Galaxy therefore relies upon the description of this faint component. Indeed, $M$ dwarfs have been employed in several Galactic studies as they carry the fundamental information on the stellar physics as well as on the galactic structure, formation, and dynamics. Moreover, $\mathrm{M}$ dwarfs are now known to host exoplanets, including super-Earth exoplanets (Bonfils et al. 2007, 2012; Udry et al. 2007). The determination of accurate fundamental parameters for $\mathrm{M}$ dwarfs has therefore relevant implications for both stellar and Galactic astronomy. Because of their intrinsic faintness and difficulties in getting homogeneous samples with respect to age and metallicity, their physics is not yet well understood.

Historically, their atmosphere has been complex to model, with the need for computed and ab initio molecular line lists that are accurate and complete with high temperatures. But for over ten years, water vapor (Partridge \& Schwenke 1997; Barber et al. 2006) and titanium oxide (Plez 1998) line lists, the two most important opacities in strength and spectral coverage, have become available and meet these conditions. Indeed, the PHOENIX model

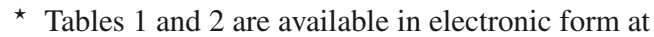
http: //www . aanda.org atmosphere synthetic spectral energy distribution (SED) has improved greatly from earlier studies Allard \& Hauschildt 1995; Hauschildt et al. 1999) to the more recent models by Allard et al. (2001, 2011, 2012a) and by Witte et al. (2011) using the most recent water vapor opacities.

The $T_{\text {eff }}$ scale of $\mathrm{M}$ dwarfs remains to this day model dependent to some level. Many efforts have been made to derive the effective temperature scale of $\mathrm{M}$ dwarfs. Due to the lack of very reliable model atmosphere, indirect methods such as blackbody fitting techniques have historically been used to estimate the effective temperature. The Bessell (1991) $T_{\text {eff }}$ scale was based on blackbody fits to the near-infrared (NIR) JHKL bands by Pettersen (1980) and Reid \& Gilmore (1984). The much cooler blackbody fits shown by Wing \& Rinsland (1979) and Veeder (1974) were fitted to the optical. Their fitting line was a continuation of the empirical $T_{\text {eff }}$ relation for the hotter stars through the Pettersen (1980) and Reid \& Gilmore (1984) NIR fits for the cooler stars. The work by Veeder (1974), Berriman \& Reid (1987), Berriman et al. (1992), and Tinney et al. (1993) also used the blackbody fitting technique to estimate the $T_{\text {eff }}$. Tsuji et al. (1996a) provide good $T_{\text {eff }}$ using infrared flux method (IRFM). Casagrande et al. (2008) provide a modified IRFM $T_{\text {eff }}$ for dwarfs including M dwarfs. These methods tend to underestimate $T_{\text {eff }}$ since the blackbody carries little flux compared to the $\mathbf{M}$ dwarfs in the Rayleigh Jeans tail redwards of $2.5 \mu \mathrm{m}$. Temperatures derived from fitting to model spectra (Kirkpatrick et al. 1993) are systematically $\sim 300 \mathrm{~K}$ warmer than 
those attained by empirical methods. This cooler $T_{\text {eff }}$ scale for M dwarfs was corrected recently by Casagrande \& Schönrich (2012) bring it close to the Bessell $(1991,1995) T_{\text {eff }}$ scale.

Tinney \& Reid (1998) determined an M dwarf $T_{\text {eff }}$ scale in the optical by ranking the objects in order of titanium oxide ( $\mathrm{TiO}$ ), vanadium oxide ( $\mathrm{VO}), \mathrm{CrH}$, and $\mathrm{FeH}$ equivalent widths. Delfosse et al. (1999) pursued a similar program in the NIR with $\mathrm{H}_{2} \mathrm{O}$ indices. Tokunaga \& Kobayashi (1999) used a spectral color index based on moderate dispersion spectroscopy in the $K$ band. Leggett et al. (1996) used observed NIR low-resolution spectra and photometry for comparison with the AMES-Dusty models (Allard et al. 2001). They found radii and effective temperatures that are consistent with the estimates based on photometric data from interior model or isochrone results. Leggett et al. (1998, 2000) revised their results by comparing the SED and NIR colors of M dwarfs to the same models. Their study provided for the first time a realistic temperature scale of M dwarfs.

In this paper, we present a new version of the BT-Settl models using the TiO line list by Plez (1998) and Plez, priv. comm., which is an important update since $\mathrm{TiO}$ accounts for the most important features in the optical spectrum. Compared to the version presented in Allard et al. (2012a) that was using Asplund et al. (2009) solar abundances, this new BT-Settl model also employs the latest solar elemental abundances by Caffau et al. (2011). We compare the revised BT-Settl synthetic spectra with the observed spectra of $152 \mathrm{M}$ dwarfs using spectral synthesis and $\chi^{2}$ minimization techniques, as well as color-color diagrams to obtain the atmospheric parameters (effective temperature, surface gravity and metallicity). We determine the revised effective temperature scale along the entire $M$ dwarfs' spectral sequence and compare these results to those obtained by many authors. Observations and spectral classification are presented in Sect. 2. Details of the model atmospheres are described in Sect. 3, and the $T_{\text {eff }}$ determination is explained in Sect. 4. The comparison between observations and models is done in Sect. 5, where spectral features and photometry are compared. The effective temperature scale of $\mathrm{M}$ dwarfs is presented in this section. Conclusions are given in Sect. 6 .

\section{Observations}

We carried out spectroscopic observations on the $3.6 \mathrm{~mm}$ New Technology Telescope (NTT) at La Silla Observatory (ESO, Chile) in November 2003. Optical low-resolution spectra were obtained in the red imaging and low-dispersion spectroscopy (RILD) observing mode with the ESO Multi Mode Instrument (EMMI) instrument. The spectral dispersion of the grism we used is $0.28 \mathrm{~nm} / \mathrm{pix}$, with a wavelength range of 385-950 $\mathrm{nm}$. We used an order-blocking filter to avoid the second-order overlap that occurs beyond $800 \mathrm{~nm}$. Thus the effective wavelength coverage ranges from 520 to $950 \mathrm{~nm}$. The slit was 1 arcsec wide and the resulting resolution was $1 \mathrm{~nm}$. The seeing varied from 0.5 to 1.5 arcsec. Exposure time ranged from $15 \mathrm{~s}$ for the brightest to $120 \mathrm{~s}$ for the faintest dwarf $(I=15.3)$. The reduction of the spectra was done using the context long of MIDAS. Fluxes were calibrated with the spectrophotometric standards LTT 2415 and Feige 110.

We obtained spectra for $97 \mathrm{M}$ dwarfs along the entire spectral sequence. They are presented in Reylé et al. (2006), Phan-Bao et al. (2005), Crifo et al. (2005), and Martín et al. (2010). The list of stars, their spectral types, and their optical and NIR photometry are given in Table 1 . The photometry has been compiled using the Vizier catalog access through the Centre de Données astronomiques de Strasbourg. It comes from the Naval Observatory Merged Astrometric Dataset (NOMAD) catalog (Zacharias et al. 2005), the Deep Near-Infrared Survey (DENIS, Epchtein 1997), and the Two Micron All Sky Survey (2MASS, Skrutskie et al. 2006), Reid et al. (2004, 2007), Koen \& Eyer (2002); Koen et al. (2010).

The observations of 55 additional M dwarfs at Siding Spring Observatory (SSO) were carried out using the Double Beam Spectrograph (DBS), which uses a dichroic beamsplitter to separate the blue (300-630 nm) and red (620-1000 nm) light. The blue camera with a $300 \mathrm{l} / \mathrm{mm}$ grating provided a 2-pixel resolution of $0.4 \mathrm{~nm}$, and the red camera with a $316 \mathrm{l} / \mathrm{mm}$ grating provided a 2-pixel resolution of $0.37 \mathrm{~nm}$. The detectors were E2V $2048 \times 51213.5$ micron/pixel CCDs. The observations were taken on Mar. 27 2008. The spectrophotometric standards used were HD 44007, HD 45282, HD 55496, HD 184266, and HD 187111 from the Next Generation Spectral Library (NGSL, version 1) $)^{1}$ L745-46a and EG131 from http://wWw.mso. anu.edu. au/ bessell/FTP/Spectrophotometry/. The list of stars with their photometry are given in Table 2.

Spectral types for the NTT sample are obtained by visual comparison with a spectral template of comparison stars, observed together with the target stars at NTT as explained in Reylé et al. (2006). For comparison, we also derive spectral types using the classification scheme based on the $\mathrm{TiO}$ and $\mathrm{CaH}$ bandstrength (Reid \& Gizis 1997). However, no comparison stars have been observed with the DBS at SSO. Thus, spectral types for the SSO sample are computed from $\mathrm{TiO}$ and $\mathrm{CaH}$ bandstrength. Although the instrument is different, we allow the comparison stars observed with EMMI on the NTT to be used as a final check. The results agree within 0.5 subclass.

\section{Model atmospheres}

For this paper, we use the most recent BT-Settl models, which are partially published in a review by Allard et al. (2012a) and described by Allard et al. (2012b). These model atmospheres are computed with the PHOENIX multi-purpose atmosphere code version 15.5 (Hauschildt et al. 1997; Allard et al. 2001) solving the radiative transfer in $1 \mathrm{D}$ spherical symmetry. The assumptions made are classical: hydrostatic equilibrium, convection using the mixing length theory, chemical equilibrium, and a sampling treatment of the opacities. The models use a mixing length as derived by the radiation hydrodynamic (RHD) simulations of Ludwig et al. (2002, 2006) and Freytag et al. (2012) and radius determined by the Baraffe et al. (1998) interior models as a function of the atmospheric parameters $\left(T_{\text {eff }}, \log g,[\mathrm{M} / \mathrm{H}]\right)$. The BTSettl grid extends from $T_{\text {eff }}=300-7000 \mathrm{~K}, \log g=2.5-5.5$, and $[\mathrm{M} / \mathrm{H}]=-2.5-0.0$ which accounts for alpha-element enrichment. The reference solar elemental abundances used in this version of the BT-Settl models are those defined by Caffau et al. (2011). The synthetic colors and spectra are distributed with a spectral resolution of around $R=100000$ via the PHOENIX web simulator ${ }^{2}$.

Hot-temperature grains have been shown to form in the uppermost layers of $\mathrm{M}$ dwarfs with effective temperatures below $3000 \mathrm{~K}$, but clear effects observable at the spectral resolution considered in this paper are only apparent below $2600 \mathrm{~K}$, that is, for later spectral types than those considered in this paper.

\footnotetext{
1 http://archive.stsci . edu/prepds/stisngsl/index.html 2 http://phoenix.ens-lyon.fr/simulator
} 

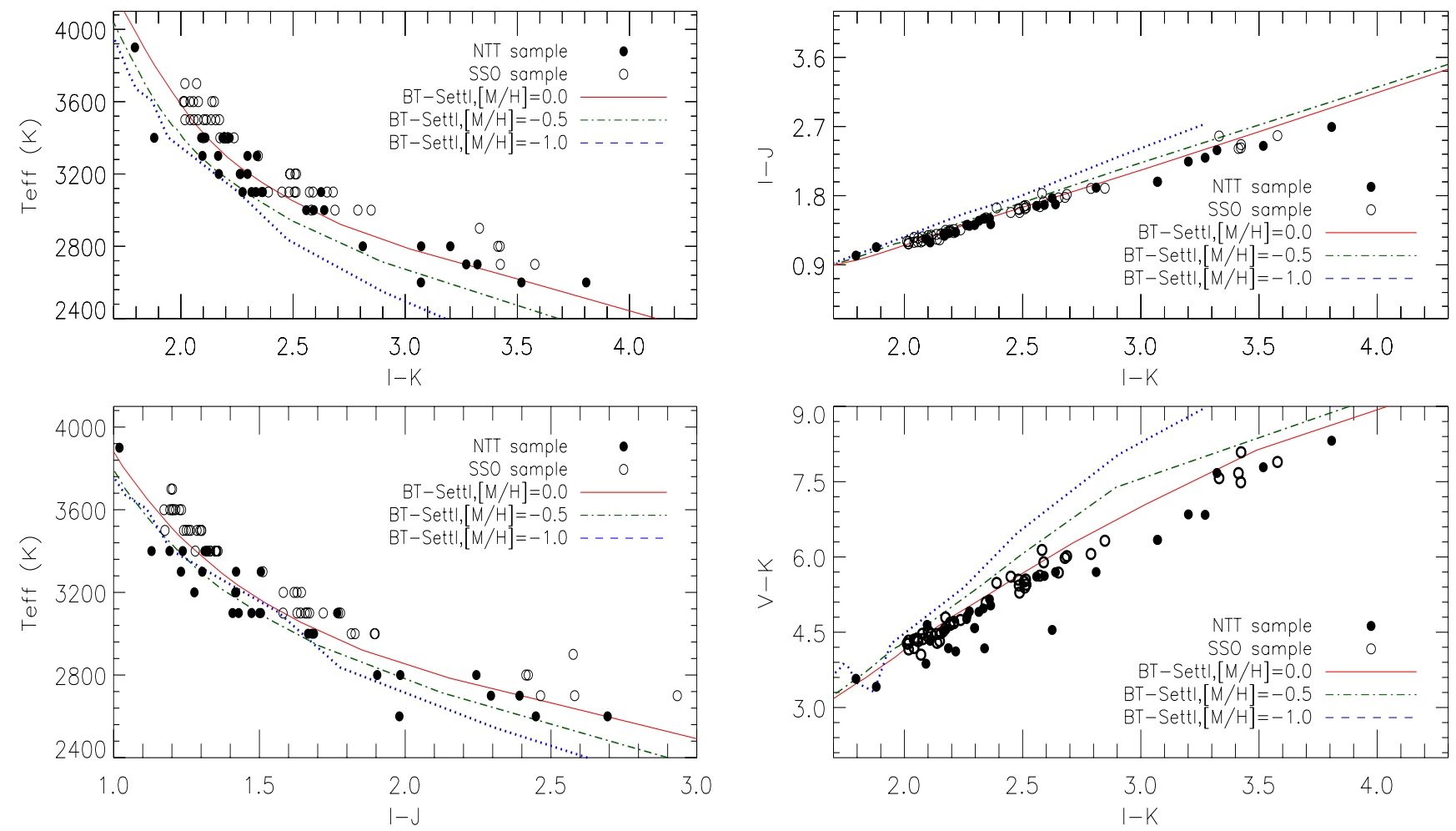

Fig. 1. $T_{\text {eff }}$ vs. NIR colors (left panel) and color-color plot (right panel) for observed M dwarfs (open and filled circle) compared to the values obtained with the $5 \mathrm{Gyr}$ isochrones from Baraffe et al. (1998) at various metallicities.

These grains produce a "veiling" by dust scattering over the optical band of the latest type M dwarfs. The BT-Settl models use therefore a slightly revised version of the Rossow (1978) cloud model (see Allard et al. 2012a,b; Rajpurohit et al. 2012b), for details on the model construction.

Compared to previous models by Allard et al. (2001), the current version of the BT-Settl model atmosphere is using the BT2 water vapor line list computed by Barber et al. (2006), TiO, VO, CaH line lists by Plez (1998), MgH by Skory et al. (2003), and Weck et al. (2003), FeH and CrH by Chowdhury et al. (2006) and Dulick et al. (2003), $\mathrm{NH}_{3}$ by Yurchenko et al. (2011), $\mathrm{CO}_{2}$ by Tashkun et al. (2004), and $\mathrm{H}_{2}$ Collision Induced Absorption (CIA) by Borysow et al. (2001) and Abel et al. (2011), to mention the most important. We use the CO line list by Goorvitch \& Chackerian (1994a,b). Detailed profiles for the alkali lines are also used (Allard et al. 2007).

In general, the Unsold (1968) approximation is used for the atomic damping constants with a correction factor to the widths of 2.5 for the non-hydrogenic atoms (Valenti \& Piskunov 1996). More accurate broadening data for neutral hydrogen collisions by Barklem et al. (2000) have been included for several important atomic transitions, such as the alkali, $\mathrm{Ca}$ I and Ca II resonance lines. For molecular lines, we adopted average values (e.g., $\left\langle\gamma_{6}^{\mathrm{HIT}}\left(T_{0}, P_{0}^{3}\right)\right\rangle_{\mathrm{H}_{2} \mathrm{O}}=0.08 P_{\text {gas }}\left[\mathrm{cm}^{-1} \mathrm{~atm}^{-1}\right]$ for water vapor lines) from the HITRAN database (Rothman et al. 2009), which are scaled to the local gas pressure and temperature:

$\gamma_{6}(T)=\left\langle\gamma_{6}^{\mathrm{HIT}}\left(T_{0}, P_{0}\right)\right\rangle\left(\frac{296 \mathrm{~K}}{T}\right)^{0.5}\left(\frac{P}{1 \mathrm{~atm}}\right)$,

with a single temperature exponent of 0.5 to be compared to values ranging mainly from 0.3 to 0.6 for water transitions studied

\footnotetext{
3 Standard temperature $296 \mathrm{~K}$ and pressure $1 \mathrm{~atm}$.
}

by Gamache et al. (1996). The HITRAN database gives widths for broadening in air, but Bailey \& Kedziora-Chudczer (2012) find that these agree in general within 10-20\% with those for broadening by a solar composition hydrogen-helium mixture.

\section{4. $T_{\text {eff }}$ determination}

We use a least-square minimization program employing the new BT-Settl model atmospheres to derive a revised effective temperature scale of M dwarfs. The stars in our samples most probably belong to the thin disc of our Galaxy (Reylé et al. 2002; Reylé $\&$ Robin 2004). Thus we determine the $T_{\text {eff }}$ of our targets by assuming solar metallicity. This is a reasonable assumption, as can be seen in Fig. 1 where we compare our two samples to three 5 Gyr isochrones with solar, $[\mathrm{M} / \mathrm{H}]=-0.5$ and $-1.0 \mathrm{dex}$. The samples are clearly compatible with solar metallicity.

Both theory and observation indicate that $\mathrm{M}$ dwarfs have $\log g=5.0 \pm 0.2$ (Gizis 1996; Casagrande et al. 2008) except for the latest type $\mathrm{M}$ dwarfs. We therefore restrict our analysis to $\log g=5.0-5.5$ models. Each synthetic spectrum was convolved to the observed spectral resolution; a scaling factor is applied to normalize the average flux to unity. We then compare each of the observed spectra with all the synthetic spectra in the grid by taking the difference between the flux values of the synthetic and observed spectra at each wavelength point. We interpolated the model spectra on the wavelength grid of the observed spectra. The sum of the squares of these differences is obtained for each model in the grid, and the best model for each object is selected. The best models were finally inspected visually by comparing them with the corresponding observed spectra. Due to the lower signal-to-noise ratio in the SSO $2.3 \mathrm{~m}$ spectra bluewards of $500 \mathrm{~nm}$ (see Fig. 3), especially for spectral types later than M4, we excluded this region below $500 \mathrm{~nm}$ from the $\chi^{2}$ computation. We also checked the variation in effective 
A\&A 556, A15 (2013)

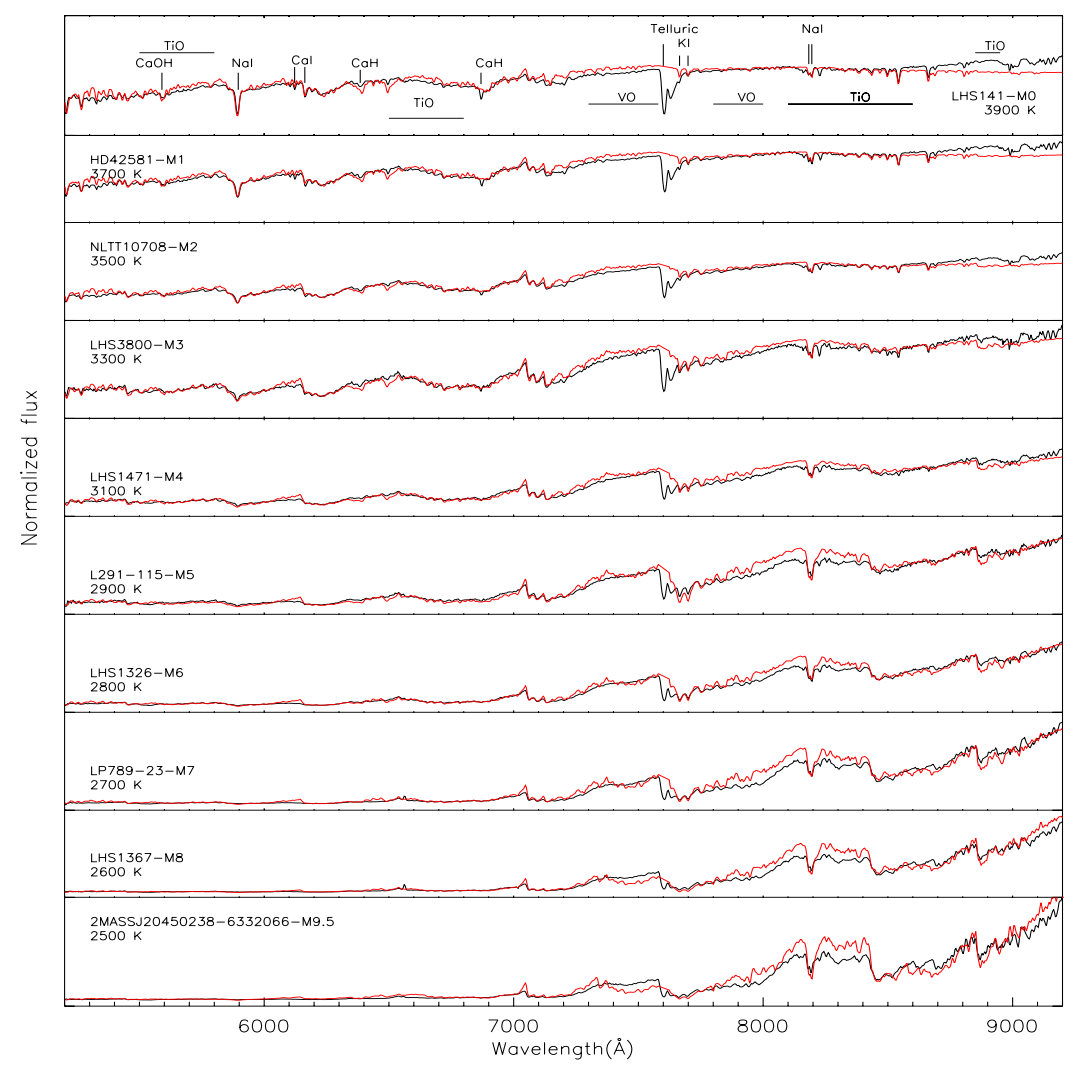

Fig. 2. Optical to red SED of M dwarfs from M0 to M9.5 observed with the NTT at a spectral resolution of $10.4 \AA$ compared to the best fit BT-Settl synthetic spectra (red lines). The models displayed have a surface gravity of $\log g=5.0$ to 5.5. Telluric features near $7600 \AA$ were ignored from the chi-square minimization.

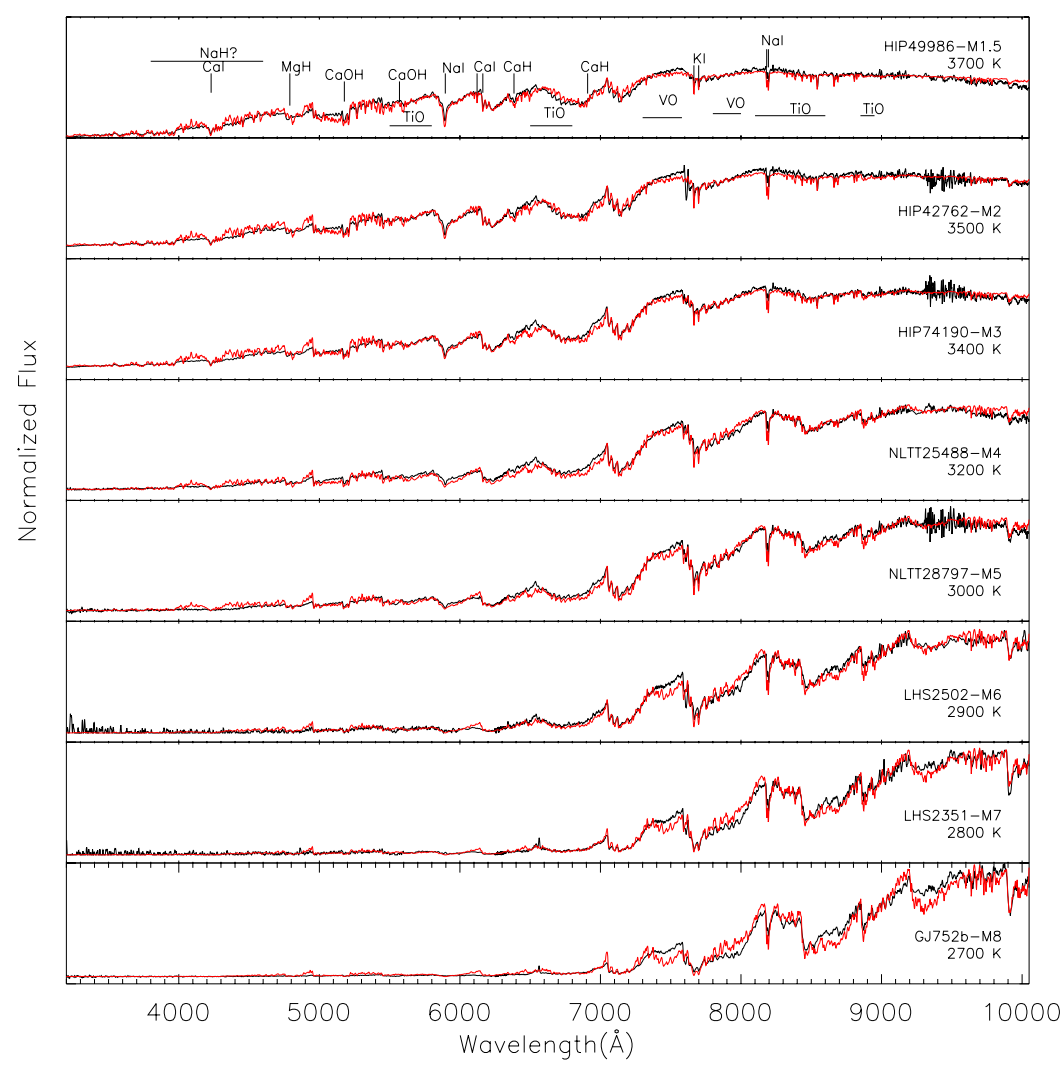

Fig. 3. Optical to red SED of M dwarfs from M1 to M8 observed with the SSO $2.3 \mathrm{~m}$ at a spectral resolution of $1.4 \AA$ compared to the best fitting (chi-square minimization) BT-Settl synthetic spectra (red lines). The models displayed have a surface gravity of $\log g=5.0$ to 5.5 . At blue wavelengths $(<5000 \AA)$ the instrumental noise dominates the late-type M dwarfs. 
temperature of the best fit as a function of the spectral type of the observed dwarfs. We found generally good agreement and conclude that our model-fitting procedure can be used to estimate the effective temperature with an uncertainty of $\sim 100 \mathrm{~K}$. The purpose of this fit is to determine the effective temperature by fitting the overall shape of the optical spectra. No attempt has been made to fit the individual atomic lines, such as the K I and $\mathrm{Na}$ I resonance doublets. With the available resolution we cannot constrain the metallicity, high-resolution spectra would be necessary (Rajpurohit et al. 2012a). In addition, we checked the influence of the spectral resolution on our derived temperatures. We degraded the resolution of the spectra of SSO $2.3 \mathrm{~m}$ down to $1 \mathrm{~nm}$ and redid the procedure. No systematic difference in $T_{\text {eff }}$ was found. The results are summarized in Tables 1 and 2.

\section{Comparison between models and observations}

\subsection{Spectroscopic confrontation}

The optical spectrum of $\mathrm{M}$ dwarfs is dominated by molecular band absorption, leaving no window onto the continuum (Allard 1990). The major opacity sources in the optical regions are due to $\mathrm{TiO}$ and $\mathrm{VO}$ bands, as well as to $\mathrm{MgH}, \mathrm{CaH}, \mathrm{FeH}$ hydride bands and $\mathrm{CAOH}$ hydroxide bands in late-type $\mathrm{M}$ dwarfs. In M dwarfs of spectral type later than M6, the outermost atmospheric layers fall below the condensation temperature of silicates, giving rise to the formation of dust clouds (Tsuji et al. 1996a,b; Allard et al. 1997).

We compared the two samples of $\mathrm{M}$ dwarfs with the most recent BT-Settl synthetic spectra in Figs. 2 and 3 through the entire $\mathrm{M}$ dwarf spectral sequence. The synthetic spectra reproduce very well the slope of the observed spectra across the $\mathrm{M}$ dwarf regime. This is a drastic improvement compared to previous comparisons of earlier models (e.g., Leggett et al. 1998).

However, some indications of missing opacities persist in the blue part of the late-type $\mathrm{M}$ dwarf, such as the $\mathrm{B}^{\prime 2} \Sigma^{+}<-X^{2} \Sigma^{+}$ system of $\mathrm{MgH}$ (Skory et al. 2003), and $\mathrm{TiO}$ and VO opacities around $8200 \AA$. Opacities are totally missing for the $\mathrm{CaOH}$ band at $5570 \AA$. The missing hydride bands of $\mathrm{AlH}$ and $\mathrm{NaH}$ between 3800 and $4600 \AA$ among others could be responsible for the remaining discrepancies. We note that chromospheric emission fills the $\mathrm{NaID}$ transitions in the latest type $\mathrm{M}$ dwarfs displayed here.

We see in this spectral regime no signs of dust scattering or of the weakening of features due to sedimentation onto grains until the M8 and later spectral types, where the spectrum becomes flat due to the sedimentation of $\mathrm{TiO}$ and $\mathrm{VO}$ bands and to the veiling by dust scattering.

\subsection{Photometric confrontation}

The models can be validated by comparing published isochrones interpolated into the new BT-Settl synthetic color tables with observed photometry. We took the $\log g$ and $T_{\text {eff }}$ for the fixed age of 5 Gyr from Baraffe et al. (1998) isochrones and calculated the colors of the star according to the BT-Settl models. The models are compared to observations in color-color diagrams in Fig. 4 for our two samples. The compiled photometry in the NTT sample is less homogeneous and translates to a larger spread, in particular for colors including the $V$ and $R$ band. This dispersion becomes dramatic for the coolest and faintest stars except for lowest mass objects at very young ages. The isochrone reproduces the two samples over the entire M dwarf spectral range in most colors. In particular, the models reproduce
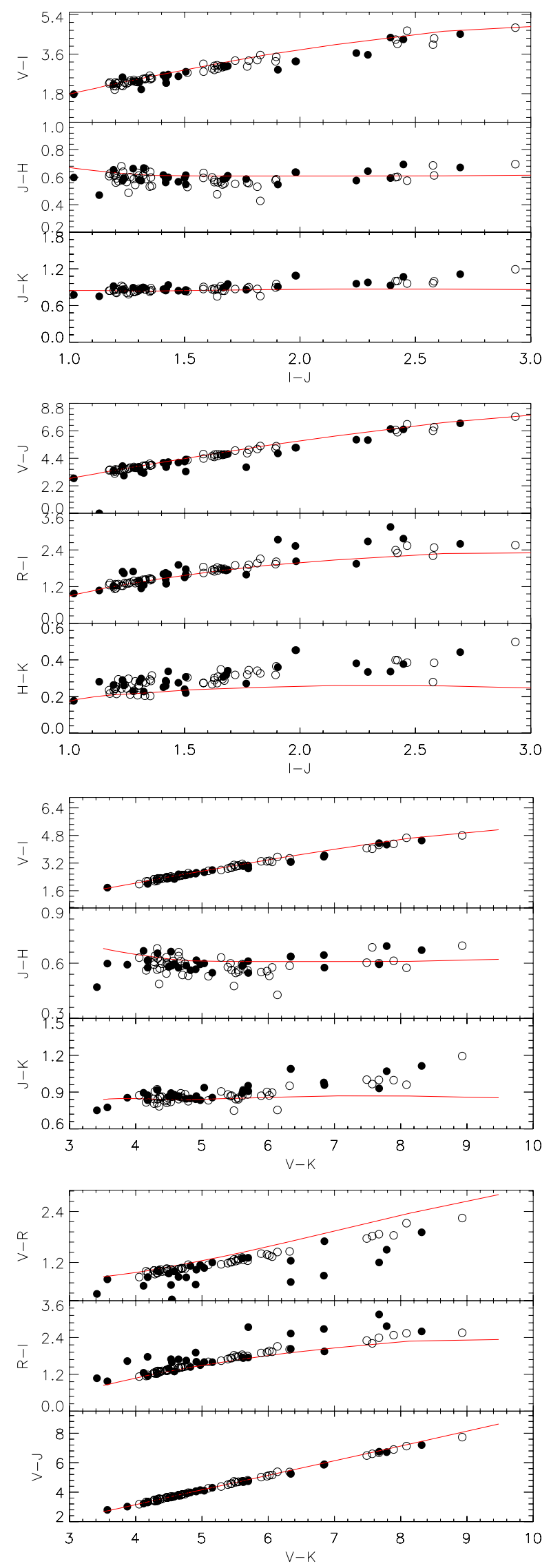

Fig. 4. Optical and NIR colors obtained with the $5 \mathrm{Gyr}$ isochrones from Baraffe et al. (1998) at solar metallicity compared with the two observation samples (filled circles for the NTT sample and open circle for the SSO $2.3 \mathrm{~m}$ spectra). Typical error bars are comparable or smaller than the size of the symbols. 


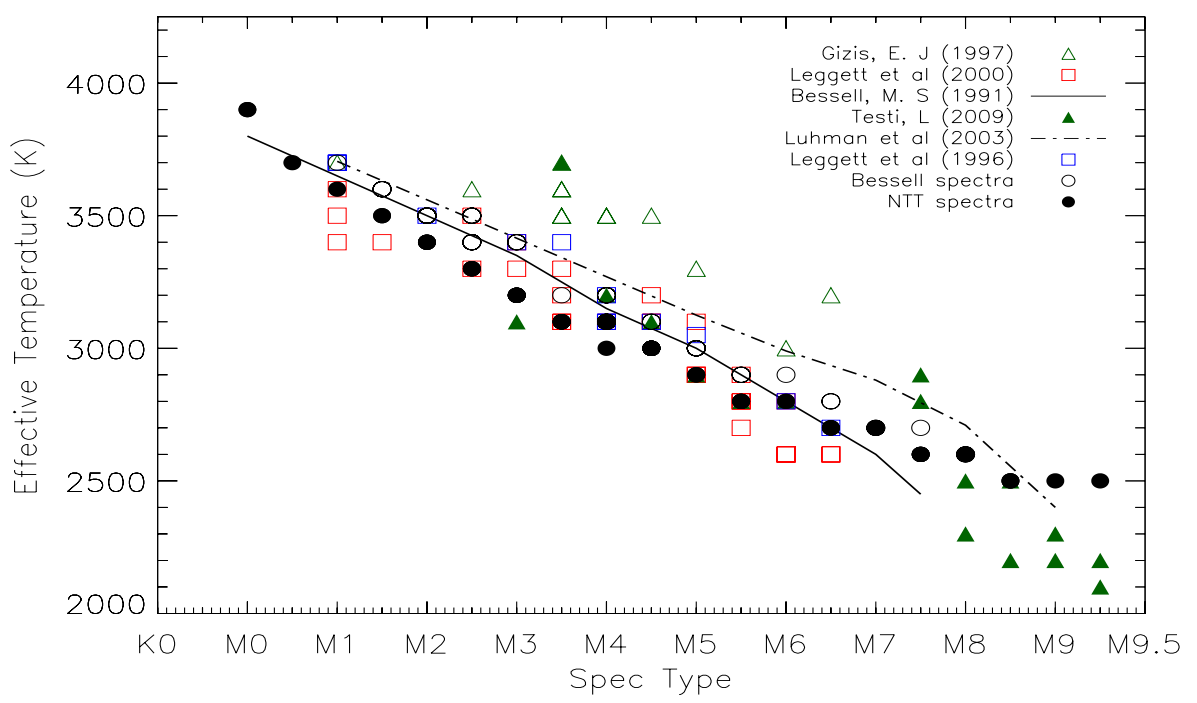

Fig. 5. Spectral type $-T_{\text {eff }}$ relation obtained with the NTT sample (filled circles) and the SSO $2.3 \mathrm{~m}$ sample (open circles) compared to relations by Bessell (1991), Gizis (1997), Leggett et al. (1996), Leggett et al. (2000), Testi (2009), and Luhman et al. (2003). the $V$-band colors of M dwarfs, as illustrated by the $V-I, V-J$, and $V-K$ colors. An increasing offset to the latest types persists in the $H-K$ and $V-R$ color indices. The observations also suggest a flattening and possibly a rise in $J-H$ and $J-K$ to the latest types, which is not reproduced by the model. These inadequacies at the coolest temperature could be linked to missing opacities.

\subsection{The $T_{\text {eff }}$ scale of $M d$ warfs}

The effective temperature scale versus spectral type is shown in Fig. 5. The $T_{\text {eff }}$ scale determined using the NTT sample (filled circles) is in agreement with the SSO sample (open circles), but we found systematically $100 \mathrm{~K}$ higher $T_{\text {eff }}$ for SSO samples for spectral types later than M5. The relation shows a saturation trend for spectral types later than M8. This illustrates the fact that the optical spectrum no longer changes sensitively with $T_{\text {eff }}$ in this regime due to dust formation.

In the following we compare our scale to other works. Bessell (1991) determined the temperatures by comparing blackbodies to the NIR photometry of their sample. They used the temperature calibration of Wing \& Rinsland (1979) and Veeder (1974). These calibrations were identical between $2700 \leq T_{\text {eff }} \leq$ $3500 \mathrm{~K}$. Their scale agrees with the modern values for M dwarfs earlier than M6, but becomes gradually too cool with later spectral types and too hot for earlier $M$ types.

Leggett et al. (1996) used the base grid by Allard \& Hauschildt (1995), which covers the range of parameters down to the coolest known M dwarfs, M subdwarfs, and brown dwarfs. They obtained the $T_{\text {eff }}$ of $\mathrm{M}$ dwarfs by comparing the observed spectra to the synthetic spectra. They performed their comparison independently at each of their four wavelength regions: red, $J, H$, and $K$. The different wavelength regions gave consistent values of $T_{\text {eff }}$ within $300 \mathrm{~K}$. Gizis (1997) used the NextGen model atmosphere grid by Allard et al. (1997). These models include more molecular lines from ab initio simulations (in particular for water vapor) than the previous base model grid. Leggett et al. (2000) used the more modern AMES-Dusty model atmosphere grid by Allard et al. (2001). They obtained a revised $T_{\text {eff }}$ scale which is $150-200 \mathrm{~K}$ cooler for early Ms and $200 \mathrm{~K}$ hotter for late Ms than the scale presented in Fig. 5. Testi (2009) determined the $T_{\text {eff }}$ by fitting the synthetic spectra to the observations. They used three classes of models: the AMES-Dusty, AMES-Cond, and the BT-Settl models. With some individual exceptions they found that the BT-Settl models were the most appropriate for $\mathrm{M}$ type and early L-type dwarfs.

Finally, for spectral types later than M0, Luhman et al. (2003) adopted the effective temperature, which is based on the NextGen and AMES-Dusty evolutionary models of Baraffe et al. (1998) and Chabrier et al. (2000), respectively. They obtained the $T_{\text {eff }}$ by comparing the H-R diagram from theoretical isochrones of Baraffe et al. (1998) and Chabrier et al. (2000). For M8 and M9, Luhman et al. (2003) adjusted the temperature scale from Luhman (1999), so that spectral sequence falls parallel to the isochrones. Their $T_{\text {eff }}$ conversion is likely to be inaccurate at some level, but as it falls between the scales for dwarfs and giants, the errors in $T_{\text {eff }}$ are modest.

The different $T_{\text {eff }}$ scales are in agreement within $250-300 \mathrm{~K}$. But the Gizis (1997) relation shows the largest differences with

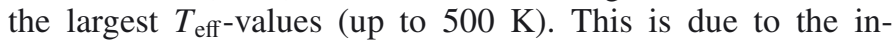
completeness of the $\mathrm{TiO}$ and water vapor line lists used in the NextGen model atmospheres. We also note also how the Luhman et al. (2003) $T_{\text {eff }}$ scale is gradually overestimating $T_{\text {eff }}$ towards the bottom of the main sequence for spectral types later than M4.

$T_{\text {eff }}$ versus color relations are shown in Fig. 6 in various photometric bands. The photometry of our NTT sample (filled circles) is compiled from the literature, which causes a large spread, particularly in the $V$ and $R$ band. The SSO $2.3 \mathrm{~m}$ sample (open circles) in comparison is more uniform. Our relations are compared to the predictions from BT-Settl isochrones at 5 Gyr. The relations show that the model is able to reproduce quite properly the colors of $\mathrm{M}$ dwarfs, even in the $V$-band. There is a slight offset visible in the $R$ band due to missing molecular opacities (see above). These relations are compared to previously published relations when available.

Berriman et al. (1992) derive the $T_{\text {eff }}$ by matching the blackbody flux anchored at $K$ band $(2.2 \mu \mathrm{m})$ to the total bolometric flux, including both the spectroscopic and photometric observed data points. They estimated the uncertainties in $T_{\text {eff }}$ to be $\pm 4 \%$. Leggett et al. (1996) used the synthetic $I-K$ and $I-J$ colors to estimate $T_{\text {eff. Leggett et al. (1996) used synthetic broadband colors }}$ from the preliminary version of AMES-Dusty model produced by Allard et al. (1994). They used the $V-K, I-K, J-H$, and $H-K$ colors assuming $\log g=5.0$ and solar metallicity, finding a hotter $T_{\text {eff }}$ scale (by on average $130 \mathrm{~K}$ ) than that of Berriman et al. (1992). More recently, Casagrande et al. (2008) used the PHOENIX Cond-GAIA model atmosphere grid (Hauschildt, unpublished) to determine the atmospheric parameters of their 
A. S. Rajpurohit et al.: The $T_{\text {eff }}$ scale of M dwarfs
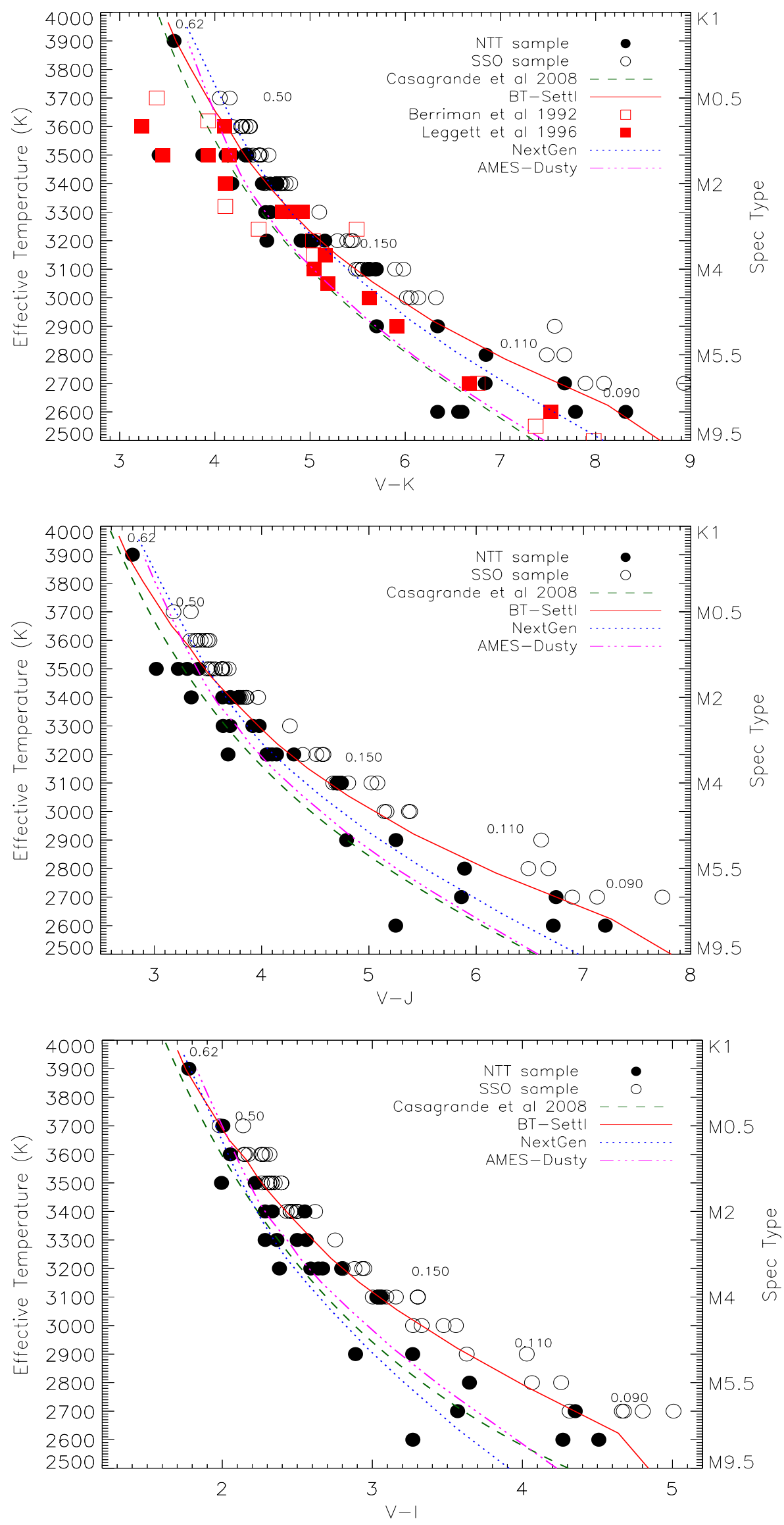

Fig. 6. Colored $T_{\text {eff }}$ plots in different bands from the NTT sample (filled circles) and the SSO $2.3 \mathrm{~m}$ sample (open circles). Spectral types are also indicated. The predictions from BT-Settl (solid line), NextGen (dotted line), and AMESDusty (dash-dotted) for solar metallicities are over plotted. Theoretical masses in solar mass are indicated. Predictions from other authors are shown for comparison when available. 
A\&A 556, A15 (2013)
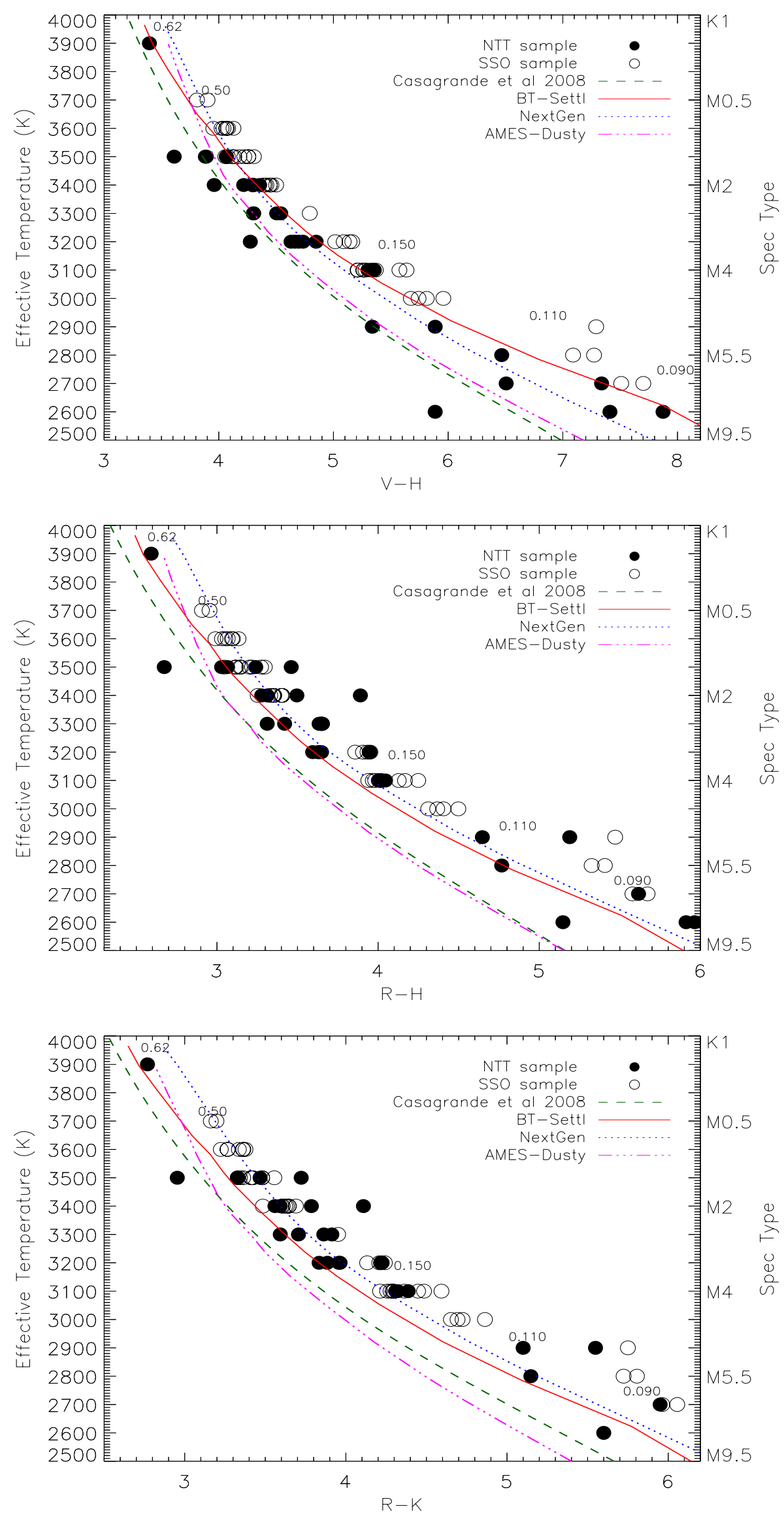

Fig. 6. continued. 
A. S. Rajpurohit et al.: The $T_{\text {eff }}$ scale of M dwarfs
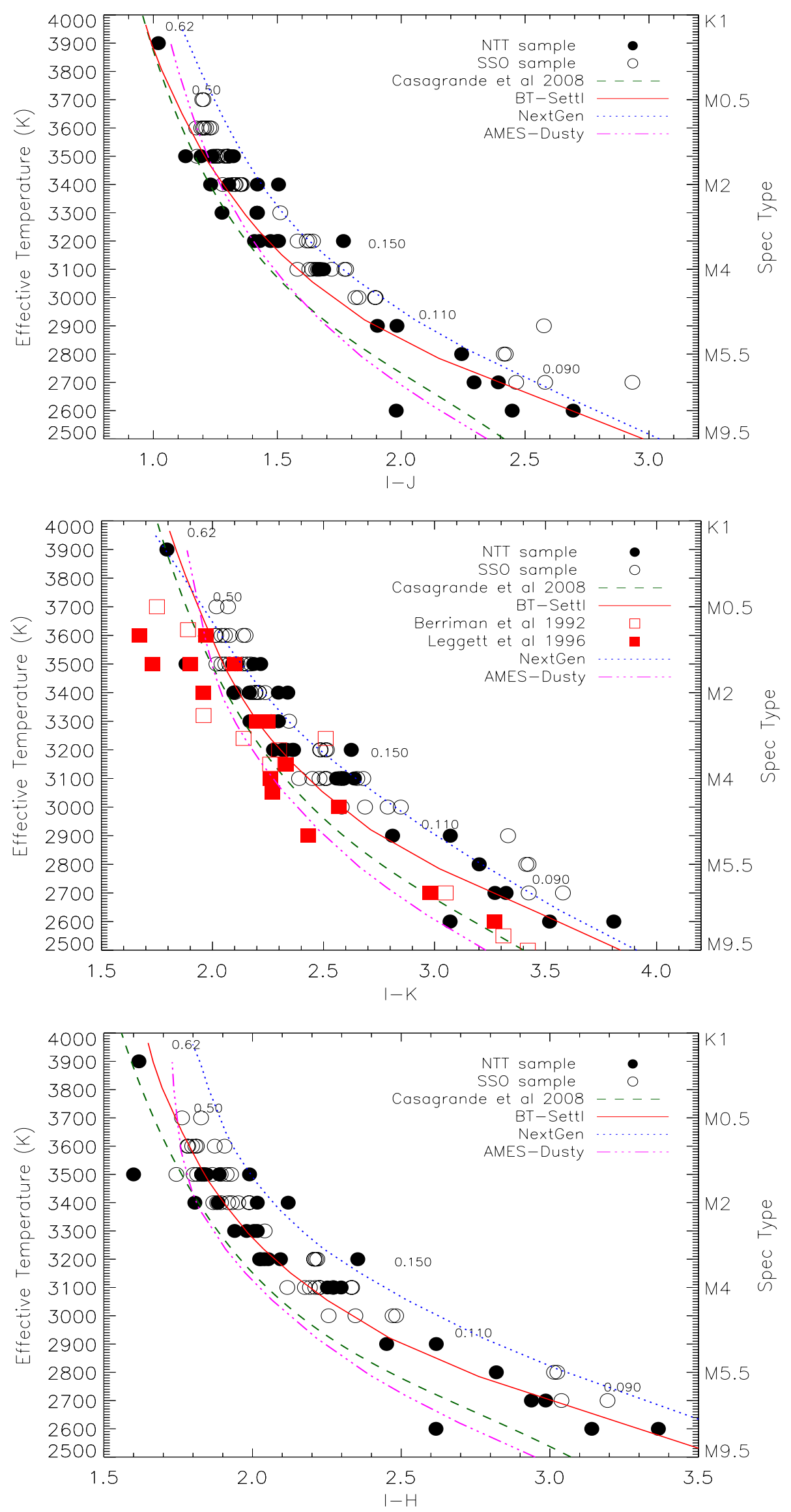

Fig. 6. continued. 
sample of 343 nearby M dwarfs with high-quality optical and NIR photometry. These models are similar to those published by Allard et al. (2001), with the exception that they were computed by solving the radiative transfer in spherical symmetry. The authors determined the $T_{\text {eff }}$ using a version of the multiple optical-infrared method (IRFM) generalized to $M$ dwarfs, and elaborated by Blackwell \& Shallis (1977) and Blackwell et al. $(1979,1980)$. Figure 6 shows that the Casagrande et al. (2008) $T_{\text {eff }}$ scale is systematically, and progressively with decreasing $T_{\text {eff }}$, cooler than the BT-Settl isochrones. Given that a large number of stars are common with Casagrande et al. (2008) sample, we did a star-by-star comparison of the $T_{\text {eff }}$ determination. The values are given in Tables 1 and 2. The comparison confirms the systematic offset in the temperature scale. For cooler stars with $T_{\text {eff }}<3000 \mathrm{~K}$, the $T_{\text {eff }}$ determinations diverge by 100 to $300 \mathrm{~K}$. This is due, among other things, to the use of the Grevesse et al. (1993) solar elemental abundances (see Allard et al. 2012, for a comparison of the different solar elemental abundance determinations and their effects on model atmospheres).

\section{Conclusion}

We have compared a revised version of the BT-Settl model atmospheres (Allard et al. 2012a) to the observed NTT and SSO $2.3 \mathrm{~m}$ spectra and colors. This new version uses the Caffau et al. (2011) solar elemental abundances, updates to the atomic and molecular line broadening, and the $\mathrm{TiO}$ line list from Plez (1998) and Plez (priv. comm.). This list provides a more accurate description of the TiO bands in the M dwarfs. The systematic discrepancy between the delta and epsilon bands found by Reiners (2005), which seriously affected the effective temperature determination, is largely alleviated by using the Plez (1998) and Plez (priv. comm.) TiO line list although discrepancies remain for the coolest stars. The BT-Settl models reproduce the SED and observed colors across the $\mathrm{M}$ dwarfs' spectral regime in unprecedented quality, as well as the colors. The $V$ band is also well reproduced by the models. Discrepancies remain in the strength of some molecular absorption bands while other absorption bands are missing, in particular in the blue spectral range.

Effective temperatures were determined by using a leastsquare minimization routine, which gives accurate temperatures within $100 \mathrm{~K}$ uncertainty. We compared our temperature color to relations using multi-wavelength photometry with the predictions from BT-Settl isochrones, assuming an age of 5 Gyr. In general, the BT-Settl isochrones are in good agreement with the observed colors, even at temperatures below $2800 \mathrm{~K}$ affected by dust treatment in the BT-Settl models. We found that the Casagrande et al. (2008) $T_{\text {eff }}$ scale is systematically cooler than the BT-Settl isochrones due, among other things, to the Grevesse et al. (1993) solar elemental abundances adopted in the GAIA-Cond model atmosphere grid used for that work. In contrast the Luhman et al. (2003) $T_{\text {eff }}$ scale is progressively too hot towards the bottom of the main sequence. New interior and evolution models based on the BT-Settl models are currently being prepared.

We provide and compare temperature versus color relations in the optical and infrared, which match well the BT-Settl isochrones and can be further used for large photometric datasets. We determined the effective temperature scale for the $\mathrm{M}$ dwarfs in our samples. It extended down to the latest type of $\mathrm{M}$ dwarfs, where the dust cloud begins to form in their atmosphere.
Acknowledgements. The use of Simbad and Vizier databases at CDS as well as the ARICNS database were very helpful for this research. The research leading to these results has received funding from the French Agence Nationale de la Recherche, the Programme National de Physique Stellaire of CNRS (INSU), and the European Research Council under the European Community's Seventh Framework Programme (FP7/2007-2013 Grant Agreement no. 247060). It was also conducted within the Lyon Institute of Origins under grant ANR-10-LABX-66. The computations were performed at the Pôle Scientifique de Modélisation Numérique (PSMN) at the École Normale Supérieure in Lyon, and at the Gesellschaft für Wissenschaftliche Datenverarbeitung Göttingen in collaboration with the Institut für Astrophysik Göttingen.

\section{References}

Abel, M., Frommhold, L., Li, X., \& Hunt, K. L. C. 2011, in 66th International Symposium On Molecular Spectroscopy

Allard, F. 1990, Ph.D. Thesis (Heidelberg: Ruprecht Karls Univ.)

Allard, F., \& Hauschildt, P. H. 1995, ApJ, 445, 433

Allard, F., Hauschildt, P. H., Miller, S., \& Tennyson, J. 1994, ApJ, 426, L39

Allard, F., Hauschildt, P. H., Alexander, D. R., \& Starrfield, S. 1997, ARA\&A, 35,137

Allard, F., Hauschildt, P. H., Alexander, D. R., Tamanai, A., \& Schweitzer, A. 2001, ApJ, 556, 357

Allard, N. F., Kielkopf, J. F., \& Allard, F. 2007, Eur. Phys. J. D, 44, 507

Allard, F., Homeier, D., \& Freytag, B. 2011, in ASP Conf. Ser., 448, eds. C. Johns-Krull, M. K. Browning, \& A. A. West, 91

Allard, F., Homeier, D., \& Freytag, B. 2012a, Roy. Soc. London Philos. Trans. Ser. A, 370, 2765

Allard, F., Homeier, D., Freytag, B., \& Sharp, C. M. 2012b, in EAS Pub. Ser., eds. C. Reylé, C. Charbonnel, \& M. Schultheis, 57, 3

Asplund, M., Grevesse, N., Sauval, A. J., \& Scott, P. 2009, ARA\&A, 47, 481

Bailey, J., \& Kedziora-Chudczer, L. 2012, MNRAS, 419, 1913

Baraffe, I., Chabrier, G., Allard, F., \& Hauschildt, P. H. 1998, A\&A, 337, 403

Barber, R. J., Tennyson, J., Harris, G. J., \& Tolchenov, R. N. 2006, MNRAS, 368,1087

Barklem, P. S., Piskunov, N., \& O’Mara, B. J. 2000, A\&A, 363, 1091

Berriman, G., \& Reid, N. 1987, MNRAS, 227, 315

Berriman, G., Reid, N., \& Leggett, S. K. 1992, ApJ, 392, L31

Bessell, M. S. 1991, AJ, 101, 662

Bessell, M. S. 1995, in The Bottom of the Main Sequence - and Beyond, ed. C. G. Tinney, 123

Blackwell, D. E., \& Shallis, M. J. 1977, MNRAS, 180, 177

Blackwell, D. E., Shallis, M. J., \& Selby, M. J. 1979, MNRAS, 188, 847

Blackwell, D. E., Petford, A. D., \& Shallis, M. J. 1980, A\&A, 82, 249

Bochanski, J. J., Hawley, S. L., Covey, K. R., et al. 2010, AJ, 139, 2679

Bonfils, X., Mayor, M., Delfosse, X., et al. 2007, A\&A, 474, 293

Bonfils, X., Gillon, M., Udry, S., et al. 2012, A\&A, 546, A27

Borysow, A., Jørgensen, U. G., \& Fu, Y. 2001, J. Quant. Spectr. Rad. Trans., 68, 235

Caffau, E., Ludwig, H.-G., Steffen, M., Freytag, B., \& Bonifacio, P. 2011, Sol. Phys., 268, 255

Casagrande, L., \& Schönrich, R. 2012, in Eur. Phys. J. Web Conf., 19, 5004

Casagrande, L., Flynn, C., \& Bessell, M. 2008, MNRAS, 389, 585

Chabrier, G. 2003, PASP, 115, 763

Chabrier, G. 2005, in The Initial Mass Function 50 Years Later, eds. E. Corbelli, F. Palla, \& H. Zinnecker, Astrophys. Space Sci. Lib., 327, 41

Chabrier, G., Baraffe, I., Allard, F., \& Hauschildt, P. 2000, ApJ, 542, 464

Chowdhury, P. K., Merer, A. J., Rixon, S. J., Bernath, P. F., \& Ram, R. S. 2006, Physical Chemistry Chemical Physics (Incorporating Faraday Transactions), 8,822

Crifo, F., Phan-Bao, N., Delfosse, X., et al. 2005, A\&A, 441, 653

Delfosse, X., Tinney, C. G., Forveille, T., et al. 1999, A\&AS, 135, 41

Dulick, M., Bauschlicher, C. W., Jr., Burrows, A., et al. 2003, ApJ, 594, 651

Epchtein, N. 1997, in The Impact of Large Scale Near-IR Sky Surveys, eds. F. Garzon, N. Epchtein, A. Omont, B. Burton, \& P. Persi, Astrophys. Space Sci. Libr., 210, 15

Freytag, B., Steffen, M., Ludwig, H.-G., et al. 2012, J. Comp. Phys., 231, 919

Gamache, R. R., Lynch, R., \& Brown, L. R. 1996, J. Quant. Spec. Radiat. Transf., 56,471

Gizis, J. E. 1996, in Cool Stars, Stellar Systems, and the Sun, eds. R. Pallavicini, \& A. K. Dupree, ASP Conf. Ser., 109, 683

Gizis, J. E. 1997, AJ, 113, 806

Goorvitch, D., \& Chackerian, C. Jr. 1994a, ApJS, 91, 483

Goorvitch, D., \& Chackerian, C. Jr. 1994b, ApJS, 92, 311

Grevesse, N., Noels, A., \& Sauval, A. J. 1993, A\&A, 271, 587 
Hauschildt, P. H., Baron, E., \& Allard, F. 1997, ApJ, 483, 390 Hauschildt, P. H., Allard, F., \& Baron, E. 1999, ApJ, 512, 377 Kirkpatrick, J. D., Kelly, D. M., Rieke, G. H., et al. 1993, ApJ, 402, 643 Koen, C., \& Eyer, L. 2002, MNRAS, 331, 45

Koen, C., Kilkenny, D., van Wyk, F., \& Marang, F. 2010, MNRAS, 403, 1949 Leggett, S. K., Allard, F., Berriman, G., Dahn, C. C., \& Hauschildt, P. H. 1996, ApJS, 104, 117

Leggett, S. K., Allard, F., \& Hauschildt, P. H. 1998, ApJ, 509, 836

Leggett, S. K., Allard, F., Dahn, C., et al. 2000, ApJ, 535, 965

Ludwig, H.-G., Allard, F., \& Hauschildt, P. H. 2002, A\&A, 395, 99

Ludwig, H.-G., Allard, F., \& Hauschildt, P. H. 2006, A\&A, 459, 599

Luhman, K. L. 1999, ApJ, 525, 466

Luhman, K. L., Stauffer, J. R., Muench, A. A., et al. 2003, ApJ, 593, 1093

Martín, E. L., Phan-Bao, N., Bessell, M., et al. 2010, A\&A, 517, A53

Partridge, H., \& Schwenke, D. W. 1997, J. Comp. Phys., 106, 4618

Pettersen, B. R. 1980, A\&A, 82, 53

Phan-Bao, N., Martín, E. L., Reylé, C., Forveille, T., \& Lim, J. 2005, A\&A, 439, L19

Plez, B. 1998, A\&A, 337, 495

Rajpurohit, A. S., Reylé, C., Schultheis, M., et al. 2012a, in SF2A-2012: Proc. Ann. meeting Fren. Soc. Astron. Astrophys., eds. S. Boissier, P. de Laverny, N. Nardetto, R. Samadi, D. Valls-Gabaud, \& H. Wozniak, 383

Rajpurohit, A. S., Reylé, C., Schultheis, M., et al. 2012b, A\&A, 545, A85

Reid, N., \& Gilmore, G. 1984, MNRAS, 206, 19

Reid, I. N., \& Gizis, J. E. 1997, AJ, 114, 1992

Reid, I. N., Cruz, K. L., Allen, P., et al. 2004, AJ, 128, 463

Reid, I. N., Cruz, K. L., \& Allen, P. R. 2007, AJ, 133, 2825

Reiners, A. 2005, Astron. Nachr., 326, 930

Reylé, C., \& Robin, A. C. 2004, A\&A, 421, 643
Reylé, C., Robin, A. C., Scholz, R.-D., \& Irwin, M. 2002, A\&A, 390, 491

Reylé, C., Scholz, R.-D., Schultheis, M., Robin, A. C., \& Irwin, M. 2006, MNRAS, 373, 705

Rossow, W. B. 1978, Icarus, 36, 1

Rothman, L. S., Gordon, I. E., Barbe, A., et al. 2009, J. Quant. Spec. Radiat. Transf., 110, 533

Skory, S., Weck, P. F., Stancil, P. C., \& Kirby, K. 2003, ApJS, 148, 599

Skrutskie, M. F., Cutri, R. M., Stiening, R., et al. 2006, AJ, 131, 1163

Tashkun, S. A., Perevalov, V. I., Teffo, J.-L., et al. 2004, Proc. SPIE, 5311, 102

Testi, L. 2009, A\&A, 503, 639

Tinney, C. G., \& Reid, I. N. 1998, MNRAS, 301, 1031

Tinney, C. G., Mould, J. R., \& Reid, I. N. 1993, AJ, 105, 1045

Tokunaga, A. T., \& Kobayashi, N. 1999, AJ, 117, 1010

Tsuji, T., Ohnaka, K., \& Aoki, W. 1996a, A\&A, 305, L1

Tsuji, T., Ohnaka, K., Aoki, W., \& Nakajima, T. 1996b, A\&A, 308, L29

Udry, S., \& Santos, N. C. 2007, ARA\&A, 45, 397

Unsold, A. 1968, Physik der Sternatmospharen, MIT besonder Berucksichtigung der Sonne

Valenti, J. A., \& Piskunov, N. 1996, A\&AS, 118, 595

Veeder, G. J. 1974, AJ, 79, 1056

Weck, P. F., Schweitzer, A., Stancil, P. C., Hauschildt, P. H., \& Kirby, K. 2003, ApJ, 584, 459

Wing, R. F., \& Rinsland, C. P. 1979, AJ, 84, 1235

Witte, S., Helling, C., Barman, T., Heidrich, N., \& Hauschildt, P. H. 2011, A\&A, 529, A44

Yurchenko, S. N., Barber, R. J., \& Tennyson, J. 2011, MNRAS, 413, 1828

Zacharias, N., Monet, D. G., Levine, S. E., et al. 2005, VizieR Online Data Catalog, I/297 
A\&A 556, A15 (2013)

Table 1. Observable and physical quantities for our sample of stars observed at NTT with EMMI.

\begin{tabular}{|c|c|c|c|c|c|c|c|c|c|c|}
\hline Name & Spectral type & $\begin{array}{l}T_{\text {eff }} \\
(\mathrm{K})\end{array}$ & $\begin{array}{r}T_{\mathrm{eff}^{b}} \\
(\mathrm{~K})\end{array}$ & $\begin{array}{c}\log g \\
\left(\mathrm{~cm} \mathrm{~s}^{-2}\right)\end{array}$ & $V$ & $R$ & $I$ & $J$ & $H$ & $K$ \\
\hline Gl143.1 ${ }^{a}$ & K7 & 3900 & - & 5.0 & 10.03 & 9.15 & - & - & - & - \\
\hline LHS141 & M0 & 3900 & - & 5.0 & 10.15 & 9.35 & 8.38 & 7.36 & 6.76 & 6.58 \\
\hline $\operatorname{LHS}_{3833^{a}}$ & M0.5 & 3800 & - & 5.0 & 10.06 & 9.33 & - & - & - & - \\
\hline $\mathrm{HD}_{42581^{a}}$ & M1 & 3700 & - & 5.0 & 8.12 & 7.16 & 6.12 & - & - & - \\
\hline $\operatorname{LHS}_{14}^{a}$ & M1.5 & 3600 & - & 5.0 & 10.04 & 9.09 & 7.99 & - & - & - \\
\hline $\mathrm{LHS65}^{a}$ & M1.5 & 3600 & 3567 & 5.0 & 10.86 & 10.31 & 10.64 & - & - & - \\
\hline L127-33 & M2 & 3500 & - & 5.0 & 14.19 & 14.04 & 12.41 & 11.17 & 10.58 & 10.32 \\
\hline NLTT10708 & M2 & 3500 & - & 5.0 & 11.16 & 10.31 & 9.17 & 7.86 & 7.28 & 6.98 \\
\hline LP831-68 & M2 & 3500 & - & 5.0 & 11.02 & 10.02 & 8.80 & 7.61 & 6.95 & 6.69 \\
\hline NLTT83-11 & M2 & 3500 & - & 5.0 & 12.90 & 12.25 & 11.00 & 9.68 & 9.01 & 8.78 \\
\hline APMPMJ0541-5349 & M2 & 3500 & - & 5.0 & 13.30 & 12.84 & 11.77 & 10.64 & 10.17 & 9.89 \\
\hline LHS1656 & $\mathrm{M} 2.5$ & 3400 & - & 5.0 & 13.30 & 12.44 & 10.75 & 9.52 & 8.94 & 8.65 \\
\hline LP763-82 & $\mathrm{M} 2.5$ & 3400 & - & 5.0 & 12.19 & 11.25 & 9.86 & 8.55 & 7.97 & 7.69 \\
\hline LP849-55 & M2.5 & 3400 & - & 5.0 & 13.32 & 13.25 & 11.48 & 9.97 & 9.36 & 9.14 \\
\hline LHS5090 & M3 & 3300 & - & 5.0 & - & 14.97 & 12.85 & 11.58 & 11.04 & 10.84 \\
\hline LHS3800 & M3 & 3300 & - & 5.0 & - & - & 12.23 & 10.93 & 10.39 & 10.15 \\
\hline LHS3842 & M3 & 3300 & - & 5.0 & 13.80 & 12.95 & 11.30 & 9.88 & 9.29 & 9.04 \\
\hline LHS1293 & M3 & 3300 & - & 5.0 & 13.65 & 12.66 & 11.36 & 9.94 & 9.35 & 9.07 \\
\hline LP994-114 & M3 & 3300 & - & 5.0 & - & 11.59 & 10.36 & 9.00 & 8.37 & 8.15 \\
\hline LTT9783 & M3 & 3300 & - & 5.0 & - & 12.11 & 10.56 & 9.17 & 8.59 & 8.34 \\
\hline LP715-39 & M3 & 3300 & 3161 & 5.0 & 12.65 & 11.53 & 10.09 & 8.67 & 8.11 & 7.82 \\
\hline LHS $1208^{a}$ & M3 & 3300 & - & 5.0 & 9.85 & 8.97 & - & - & - & - \\
\hline LEHPM4417 & M3 & 3300 & - & 5.0 & 13.73 & 13.06 & 11.37 & 10.09 & 9.43 & 9.20 \\
\hline LP831-45 & M3.5 & 3200 & 3125 & 5.0 & 12.54 & 11.51 & 9.90 & 8.49 & 7.88 & 7.62 \\
\hline 2MASS J04060688-0534444 & M3.5 & 3200 & - & 5.0 & 13.29 & 12.28 & - & 9.13 & 8.55 & 8.30 \\
\hline LP834-32 & M3.5 & 3200 & 3108 & 5.0 & 12.38 & 11.24 & 9.74 & 8.24 & 7.65 & 7.41 \\
\hline $\mathrm{LHS} 02^{a}$ & M3.5 & 3200 & - & 5.0 & 11.49 & 10.43 & 9.11 & - & - & - \\
\hline LEHPM 1175 & M3.5 & 3200 & - & 5.0 & - & 13.08 & 11.51 & 10.01 & 9.47 & 9.17 \\
\hline LEHPM1839 & M3.5 & 3200 & - & 5.0 & - & 13.32 & 12.11 & 10.55 & 9.95 & 9.71 \\
\hline L130-37 & M3.5 & 3200 & - & 5.0 & 13.04 & 11.97 & 10.37 & 8.94 & 8.34 & 8.01 \\
\hline LEHPM6577 & M3.5 & 3200 & - & 5.0 & - & 13.03 & 11.79 & 10.34 & 9.73 & 9.47 \\
\hline L225-57 & M4 & 3200 & - & 5.0 & - & 11.70 & 9.79 & 8.23 & 7.61 & 7.31 \\
\hline LP942-107 & M4 & 3200 & 3052 & 5.0 & 13.93 & 12.73 & 11.13 & 9.63 & 9.08 & 8.77 \\
\hline LP772-8 & M4 & 3200 & - & 5.0 & 14.11 & 13.43 & 11.52 & 10.05 & 9.48 & 9.20 \\
\hline LP1033-31 & M4 & 3200 & - & 5.0 & - & 12.12 & 10.54 & 9.10 & 8.46 & 8.21 \\
\hline L166-3 & M4 & 3200 & - & 5.0 & - & 12.76 & 11.33 & 9.83 & 9.28 & 9.00 \\
\hline LP877-72 & M4 & 3200 & - & 5.0 & - & 11.- & 10.22 & 8.86 & 8.24 & 8.00 \\
\hline LP878-73 & M4 & 3200 & - & 5.0 & 14.55 & 14.22 & 12.63 & 10.86 & 10.27 & 10.00 \\
\hline LP987-47 & M4 & 3200 & - & 5.0 & - & - & 10.82 & 9.41 & 8.78 & 8.55 \\
\hline LP832-7 & M4 & 3200 & - & 5.0 & 14.09 & 13.45 & - & 9.87 & 9.24 & 8.98 \\
\hline LHS183 & M4 & 3200 & - & 5.0 & 12.79 & 11.51 & - & 8.57 & 8.00 & 7.75 \\
\hline LHS1471 & M4 & 3200 & - & 5.0 & - & 13.22 & 11.56 & 9.94 & 9.37 & 9.08 \\
\hline APMPMJ2101-4125 & M4 & 3200 & - & 5.0 & - & 13.34 & 11.47 & 9.96 & 9.38 & 9.09 \\
\hline APMPMJ2101-4907 & M4 & 3200 & - & 5.0 & - & - & 10.52 & 9.12 & 8.48 & 8.19 \\
\hline LEHPM3260 & M4 & 3200 & - & 5.0 & - & 12.53 & 10.60 & 9.13 & 8.54 & 8.19 \\
\hline LEHPM3866 & M4 & 3200 & - & 5.0 & - & - & 11.82 & 10.21 & 9.58 & 9.29 \\
\hline LEHPM5810 & M4 & 3200 & - & 5.0 & - & 13.58 & 11.66 & 9.91 & 9.33 & 9.05 \\
\hline LHS5045 & M4.5 & 3100 & - & 5.0 & - & - & 10.78 & 9.17 & 8.60 & 8.24 \\
\hline LP940-20 & M4.5 & 3100 & - & 5.0 & - & 14.87 & 12.65 & 10.92 & 10.32 & 10.01 \\
\hline L170-14A & M4.5 & 3100 & - & 5.0 & - & 12.86 & 11.50 & 9.76 & 9.13 & 8.88 \\
\hline
\end{tabular}

Notes. ${ }^{(a)}$ Saturation in NIR bands. ${ }^{(b)} T_{\text {eff }}$ from Casagrande et al. (2008). 
Table 1. continued.

\begin{tabular}{|c|c|c|c|c|c|c|c|c|c|c|}
\hline Name & Spectral type & $\begin{array}{l}T_{\text {eff }} \\
(\mathrm{K})\end{array}$ & $\begin{array}{l}T_{\mathrm{eff}^{b}} \\
(\mathrm{~K})\end{array}$ & $\begin{array}{c}\log g \\
\left(\mathrm{~cm} \mathrm{~s}^{-2}\right)\end{array}$ & $V$ & $R$ & $I$ & $J$ & $H$ & $K$ \\
\hline LHS1201 & M4.5 & 3100 & - & 5.0 & 17.55 & 15.52 & 12.90 & 11.12 & 10.52 & 10.25 \\
\hline LHS1524 & M4.5 & 3100 & - & 5.0 & - & 14.45 & 12.65 & 10.98 & 10.45 & 10.17 \\
\hline LTT1732 & M4.5 & 3100 & - & 5.0 & - & 13.19 & 11.27 & 9.69 & 9.11 & 8.80 \\
\hline LP889-37 & M4.5 & 3100 & 2923 & 5.0 & 14.52 & 13.21 & 11.46 & 9.77 & 9.16 & 8.82 \\
\hline LHS5094 & M4.5 & 3100 & - & 5.0 & 14.02 & 12.72 & 10.97 & 9.30 & 8.72 & 8.41 \\
\hline LP655-43 & M4.5 & 3100 & 2924 & 5.0 & 14.44 & 13.14 & 11.41 & 9.73 & 9.14 & 8.82 \\
\hline 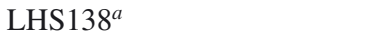 & M4.5 & 3100 & - & 5.0 & 12.07 & 10.70 & 8.94 & - & - & - \\
\hline APMPMJ1932-4834 & M4.5 & 3100 & - & 5.0 & - & 14.38 & 12.37 & 10.63 & 10.02 & 9.72 \\
\hline 2MASS J23522756-3609128 & M4.5 & 3100 & - & 5.0 & - & 17.27 & - & 13.09 & 12.57 & 12.28 \\
\hline LEHPM640 & M4.5 & 3100 & - & 5.0 & 17.74 & 14.26 & 12.30 & 10.76 & 10.14 & 9.90 \\
\hline LEHPM1853 & M4.5 & 3100 & - & 5.0 & - & 12.77 & 11.03 & 9.46 & 8.85 & 8.61 \\
\hline LEHPM3115 & M4.5 & 3100 & - & 5.0 & - & 13.94 & 12.10 & 10.49 & 9.92 & 9.63 \\
\hline LEHPM4771 & M4.5 & 3100 & - & 5.0 & 17.74 & 13.79 & 11.29 & 9.54 & 8.95 & 8.63 \\
\hline LEHPM4861 & M4.5 & 3100 & - & 5.0 & - & 13.28 & 11.75 & 10.13 & 9.60 & 9.34 \\
\hline L291-115 & M5 & 2900 & - & 5.0 & 15.88 & 14.90 & 12.26 & 10.44 & 9.83 & 9.54 \\
\hline LP904-51 & M5 & 2900 & - & 5.0 & - & 15.32 & 12.84 & 11.04 & 10.44 & 10.16 \\
\hline LHS168 & M5 & 2900 & - & 5.0 & 13.78 & 12.60 & - & 8.77 & 8.21 & 7.83 \\
\hline LP829-41 & M5.5 & 2800 & - & 5.0 & 16.10 & 15.95 & 13.21 & 11.31 & 10.76 & 10.40 \\
\hline LP941-57 & M5.5 & 2800 & - & 5.0 & - & 14.88 & 12.98 & 11.06 & 10.47 & 10.13 \\
\hline LHS546 & M5.5 & 2800 & - & 5.0 & 14.69 & - & - & 9.15 & 8.50 & 8.18 \\
\hline LP714-37 & M5.5 & 2800 & - & 5.5 & 16.26 & 15.02 & 12.99 & 11.01 & 10.37 & 9.92 \\
\hline LHS1326 & M6 & 2800 & - & 5.5 & 15.61 & 14.49 & - & 9.84 & 9.25 & 8.93 \\
\hline 2MASS J12363959-1722170 & M6 & 2800 & - & 5.0 & 17.56 & 15.86 & 13.91 & 11.67 & 11.09 & 10.71 \\
\hline 2MASS J21481595-1401059 & M6.5 & 2700 & - & 5.0 & - & 20.20 & 17.15 & 14.68 & 14.11 & 13.65 \\
\hline 2MASS J05181131-3101519 & M6.5 & 2700 & - & 5.0 & 17.74 & 16.85 & 14.17 & 11.88 & 11.23 & 10.90 \\
\hline LP788-1 & M6.5 & 2700 & - & 5.0 & - & 16.66 & 13.36 & 11.07 & 10.47 & 10.07 \\
\hline APMPMJ1251-2121 & M6.5 & 2700 & - & 5.0 & - & 16.65 & 13.78 & 11.16 & 10.55 & 10.13 \\
\hline APMPMJ2330-4737 & M7 & 2700 & - & 5.0 & - & - & 13.70 & 11.23 & 10.64 & 10.28 \\
\hline LP789-23 & M7 & 2700 & - & 5.0 & - & 17.90 & 14.55 & 12.04 & 11.39 & 10.99 \\
\hline LHS292 & M7 & 2700 & - & 5.5 & 15.60 & 14.40 & 11.25 & 8.86 & 8.26 & 7.93 \\
\hline 2MASS J03144011-0450316 & M7.5 & 2600 & - & 5.0 & - & 19.43 & - & 12.64 & 12.00 & 11.60 \\
\hline LHS1604 & M7.5 & 2600 & - & 5.0 & 18.02 & 16.52 & 13.75 & 11.30 & 10.61 & 10.23 \\
\hline LP714-37 & M7.5 & 2600 & - & 5.5 & 16.26 & 15.52 & 12.99 & 11.01 & 10.37 & 9.92 \\
\hline LP655-48 & M7.5 & 2600 & 2250 & 5.0 & 17.86 & 15.95 & 13.35 & 10.66 & 9.99 & 9.54 \\
\hline LP851-346 & M7.5 & 2600 & - & 5.5 & - & 16.79 & 13.77 & 10.93 & 10.29 & 9.88 \\
\hline LHS1367 & M8 & 2600 & - & 5.0 & - & 17.34 & 14.18 & 11.62 & 10.95 & 10.54 \\
\hline 2MASS J05022640-0453583 & M8 & 2600 & - & 5.0 & - & 20.39 & 17.35 & 14.52 & 13.95 & 13.58 \\
\hline LHS132 & M8 & 2600 & - & 5.0 & - & 17.14 & 13.83 & 11.13 & 10.48 & 10.07 \\
\hline 2MASS J22062280-2047058 & M8 & 2600 & - & 5.0 & - & 18.93 & 15.09 & 12.37 & 11.69 & 11.31 \\
\hline 2MASS J22264440-7503425 & M8 & 2600 & - & 5.0 & - & 18.95 & 15.20 & 12.35 & 11.70 & 11.25 \\
\hline 2MASS J04103617-1459269 & M8.5 & 2500 & - & 5.5 & - & - & 16.68 & 13.94 & 13.24 & 12.81 \\
\hline 2MASS J05084947-1647167 & M8.5 & 2500 & - & 5.5 & - & - & 16.46 & 13.69 & 12.96 & 12.53 \\
\hline 2MASS J04362788-4114465 & M8.5 & 2500 & - & 5.5 & - & 19.96 & 16.04 & 13.10 & 12.43 & 12.05 \\
\hline 2MASS J10481463-3956062 & M9 & 2500 & - & 5.5 & - & 15.93 & 12.67 & 9.54 & 8.90 & 8.45 \\
\hline 2MASS J20450238-6332066 & M9.5 & 2500 & - & 5.5 & - & 19.24 & 16.05 & 12.62 & 11.81 & 11.21 \\
\hline 2MASS J09532126-1014205 & M9.5 & 2500 & - & 5.5 & - & 19.58 & 16.82 & 13.47 & 12.64 & 12.14 \\
\hline
\end{tabular}


A\&A 556, A15 (2013)

Table 2. Observable and physical quantities for our sample of stars observed at SSO.

\begin{tabular}{|c|c|c|c|c|c|c|c|c|c|c|}
\hline Name & Spectral type & $\begin{array}{l}T_{\text {eff }} \\
(\mathrm{K})\end{array}$ & $\begin{array}{l}T_{\mathrm{eff}^{b}} \\
(\mathrm{~K})\end{array}$ & $\begin{array}{c}\log g \\
\left(\mathrm{~cm} \mathrm{~s}^{-2}\right)\end{array}$ & V & $R$ & $I$ & $J$ & $H$ & $K$ \\
\hline HIP 49986 & M1.5 & 3700 & 3445 & 5.0 & 9.07 & 8.21 & 7.08 & 5.89 & 5.26 & 5.01 \\
\hline HIP 82256 & M1.5 & 3700 & 3470 & 5.0 & 11.38 & 10.39 & 9.24 & 8.04 & 7.48 & 7.22 \\
\hline HIP 56528 & M1.5 & 3600 & 3472 & 5.0 & 9.81 & 8.85 & 7.66 & 6.47 & 5.86 & 5.62 \\
\hline NLTT19190 & M1.5 & 3600 & 3456 & 5.0 & 11.49 & 10.57 & 9.34 & 8.11 & 7.47 & 7.20 \\
\hline NLTT42523 & M2 & 3600 & 3444 & 5.0 & 12.08 & 11.06 & 9.81 & 8.60 & 8.01 & 7.80 \\
\hline HIP 80229 & M2 & 3600 & 3486 & 5.0 & 11.91 & 10.90 & 9.65 & 8.48 & 7.87 & 7.64 \\
\hline LP725-25 & M2 & 3600 & 3476 & 5.0 & 11.76 & 10.82 & 9.59 & 8.36 & 7.68 & 7.44 \\
\hline HIP 61413 & M2 & 3500 & 3454 & 5.0 & 11.49 & 10.48 & 9.17 & 7.99 & 7.37 & 7.15 \\
\hline LP853-34 & M2 & 3500 & 3339 & 5.0 & 12.32 & 11.31 & 9.99 & 8.69 & 8.10 & 7.83 \\
\hline LP859-11 & M2 & 3500 & 3433 & 5.0 & 12.00 & 10.97 & 9.69 & 8.49 & 7.88 & 7.63 \\
\hline LP788-49 & M2 & 3500 & 3356 & 5.0 & 11.81 & 10.85 & 9.55 & 8.30 & 7.74 & 7.49 \\
\hline HIP 42762 & M2 & 3500 & 3302 & 5.0 & 11.75 & 10.76 & 9.42 & 8.12 & 7.49 & 7.28 \\
\hline HIP 51317 & M2 & 3500 & 3403 & 5.0 & 9.67 & 8.67 & 7.34 & 6.18 & 5.60 & 5.31 \\
\hline HIP 60559 & M2 & 3500 & 3382 & 5.0 & 11.30 & 10.29 & 8.99 & 7.73 & 7.25 & 6.95 \\
\hline HIP 47103 & M2 & 3500 & 3319 & 5.0 & 10.87 & 9.89 & 8.58 & 7.34 & 6.74 & 6.47 \\
\hline HIP 93206 & M2.5 & 3500 & 3366 & 5.0 & 11.23 & 10.18 & 8.80 & 7.52 & 6.93 & 6.70 \\
\hline LP834-3 & M2.5 & 3500 & - & 5.0 & - & - & - & - & - & - \\
\hline HIP 84521 & M2.5 & 3500 & 3345 & 5.0 & 11.57 & 10.53 & 9.22 & 7.93 & 7.39 & 7.11 \\
\hline HIP 91430 & M2.5 & 3500 & 3352 & 5.0 & 11.32 & 10.26 & 8.92 & 7.66 & 7.06 & 6.85 \\
\hline HIP 50341 & M2.5 & 3500 & 3314 & 5.0 & 11.02 & 10.01 & 8.62 & 7.32 & 6.71 & 6.45 \\
\hline LP672-2 & $\mathrm{M} 2.5$ & 3400 & - & 5.0 & 12.58 & 11.54 & 10.12 & 8.80 & 8.14 & 7.93 \\
\hline NLTT24892 & M2.5 & 3400 & 3244 & 5.0 & 12.52 & 11.47 & 10.05 & 8.73 & 8.118 & 7.84 \\
\hline NLTT34577 & M2.5 & 3400 & 3254 & 5.0 & 12.44 & 11.40 & 9.99 & 8.64 & 8.00 & 7.80 \\
\hline LP670-17 & M3 & 3400 & 3226 & 5.0 & 12.14 & 11.08 & 9.63 & 8.28 & 7.68 & 7.39 \\
\hline HIP 59406 & M3 & 3400 & 3226 & 5.0 & 11.75 & 10.69 & 9.25 & 7.89 & 7.36 & 7.04 \\
\hline HIP 74190 & M3 & 3400 & 3258 & 5.0 & 11.55 & 10.48 & 9.05 & 7.72 & 7.13 & 6.86 \\
\hline NLTT46868 & M3.5 & 3400 & 3221 & 5.0 & 12.23 & 11.08 & 9.61 & 8.26 & 7.73 & 7.44 \\
\hline HIP 62452 & M4 & 3300 & 3095 & 5.0 & 11.46 & 10.31 & 8.71 & 7.19 & 6.67 & 6.36 \\
\hline NLTT25488 & M4 & 3200 & 2986 & 5.0 & 15.66 & 14.46 & 12.73 & 11.09 & 10.52 & 10.21 \\
\hline NLTT29087 & M4 & 3200 & 2971 & 5.0 & 14.79 & 13.57 & 11.84 & 10.22 & 9.62 & 9.35 \\
\hline NLTT29790 & M4 & 3200 & 2987 & 5.0 & 14.73 & 13.54 & 11.85 & 10.22 & 9.64 & 9.34 \\
\hline LP734-32 & M4 & 3200 & 3024 & 5.0 & 12.15 & 10.99 & 9.35 & 7.77 & 7.14 & 6.86 \\
\hline LP739-2 & M4 & 3100 & 2939 & 5.0 & 14.44 & 13.18 & 11.40 & 9.73 & 9.17 & 8.89 \\
\hline LP735-29 & M4 & 3100 & 2940 & 5.0 & 14.18 & 12.95 & 11.18 & 9.52 & 8.97 & 8.67 \\
\hline GJ1123 & M4 & 3100 & - & 5.0 & 13.14 & 11.90 & 10.10 & 8.33 & 7.77 & 7.45 \\
\hline GJ1128 & M4 & 3100 & - & 5.0 & 12.66 & 11.40 & 9.61 & 7.95 & 7.38 & 7.04 \\
\hline NLTT35266 & M4.5 & 3100 & 2942 & 5.0 & 15.15 & 13.88 & 12.05 & 10.41 & 9.94 & 9.66 \\
\hline NLTT41951 & M4.5 & 3100 & & 5.0 & 15.06 & 13.77 & 11.99 & 10.36 & 9.80 & 9.51 \\
\hline NLTT21329 & M4.5 & 3000 & 2949 & 5.0 & 13.75 & 12.38 & 10.42 & 8.60 & 8.07 & 7.73 \\
\hline LP732-35 & M5 & 3100 & 2901 & 5.0 & 14.10 & 12.78 & 10.94 & 9.36 & 8.76 & 8.49 \\
\hline NLTT18930 & M5 & 3100 & 2903 & 5.0 & 15.34 & 13.93 & 12.03 & 10.31 & 9.76 & 9.44 \\
\hline 2MASS J14221943-7023371 & M5 & 3000 & - & 5.0 & - & - & - & - & - & - \\
\hline NLTT22503 & M5 & 3000 & 2785 & 5.0 & 13.66 & 12.32 & 10.39 & 8.50 & 7.92 & 7.60 \\
\hline NLTT28797 & M5 & 3000 & 2826 & 5.0 & 15.62 & 14.24 & 12.32 & 10.54 & 9.99 & 9.64 \\
\hline NLTT30693 & M5.5 & 3000 & 2785 & 5.5 & 15.32 & 13.86 & 11.85 & 9.95 & 9.36 & 9.00 \\
\hline LHS288 & M5.5 & 3000 & 2770 & 5.0 & 13.87 & 12.42 & 10.31 & 8.48 & 8.05 & 7.73 \\
\hline GJ551 & M5.5 & 2900 & - & 5.0 & 3.63 & 2.08 & 5.36 & 4.83 & 4.38 & - \\
\hline LHS2502 & M6 & 2900 & 2468 & 5.5 & 19.36 & 17.54 & 15.33 & 12.75 & 12.07 & 11.79 \\
\hline NLTT20726 & M6.5 & 2800 & 2464 & 5.0 & 16.11 & 14.24 & 11.85 & 9.44 & 8.84 & 8.44 \\
\hline GJ406 & M6.5 & 2800 & - & 5.5 & 13.57 & 11.81 & 9.51 & 7.08 & 6.48 & 6.08 \\
\hline LHS2351 & M7 & 2800 & 2346 & 5.5 & 19.22 & 17.39 & 14.91 & 12.33 & 11.72 & 11.33 \\
\hline SCR J1546-5534 & M7.5 & 2700 & - & 5.5 & - & - & - & - & - & - \\
\hline GJ752b & M8 & 2700 & - & 5.5 & 5.01 & - & - & 9.91 & 9.23 & 8.76 \\
\hline GJ644c & M7 & 2700 & - & 5.5 & 16.90 & 14.78 & 12.24 & 9.78 & 9.20 & 8.82 \\
\hline LHS2397a & M8 & 2700 & - & 5.5 & 19.66 & 17.42 & 14.86 & 11.93 & 11.23 & 10.73 \\
\hline
\end{tabular}

Notes. ${ }^{(b)} T_{\text {eff }}$ from Casagrande et al. (2008). 